\title{
Tissue Doppler echocardiography can be a useful technique to evaluate atrial conduction time
}

\author{
Ali Deniz, Levent Sahiner, Kudret Aytemir, Baris Kaya, \\ Giray Kabakci, Lale Tokgozoglu, Ali Oto \\ Department of Cardiology, Faculty of Medicine, Hacettepe University, Ankara, Turkey
}

\begin{abstract}
Background: The main purpose of this study is to determine the correlation of inter-and intraatrial conduction times between the electrophysiological and tissue Doppler echocardiographic measurements, and to evaluate the appropriateness of tissue Doppler echocardiography for this measurement.

Methods: One-hundred and one patients were included in the study who underwent electrophysiological study for clinical arrhythmias. Inter-and intraatrial conduction times were measured from intracardiac electrograms. Atrial conduction times were also measured by tissue Doppler echocardiography by evaluating atrial electromechanical delay between lateral mitral annulus, septal mitral annulus, and right ventricular tricuspid annulus. The correlation between electrophysiological and echocardiographic atrial conduction times were analyzed.

Results: We found a weak correlation between the measurements of interatrial conduction times with the electrophysiological and tissue Doppler techniques $(r=0.308 ; p=0.002)$. The correlation for intraleft atrial conduction times was moderate $(r=0.652 ; p<0.001)$. There was no correlation between the measurements of intra-right atrial conduction times.

Conclusions: We concluded that tissue Doppler echocardiography can be used for the measurement of interatrial and intra-left atrial conduction times. Tissue Doppler echocardiography can be a suitable technique to evaluate atrial substrate. (Cardiol J 2012; 19, 5: 487-493)
\end{abstract}

Key words: atrial conduction time, tissue Doppler echocardiography

\section{Introduction}

Conduction of sinus beat through the atria requires electrical and functional continuity of atrial myocytes. Atrial conduction time which is inversely related to atrial conduction velocity is a measure of atrial conduction characteristics. Any pathologic process impairing atrial conduction may result in reentrant atrial arrhythmias. Atrial fibrillation (AF) is one of the most common arrhythmias observed in clinical practice [1]. Interatrial conduction abnormalities may result in initiation and continuation of
$\mathrm{AF}[2,3]$. The frequency of $\mathrm{AF}$ is increased in patients with impaired interatrial conduction [4]. It has been shown that in the presence of interatrial conduction delay, biatrial, bifocal right atrial, and interatrial septal pacing may prevent the recurrence of drug-resistant paroxysmal AF [5-7].

Among the noninvasive and invasive methods to evaluate the interatrial conduction, the basic and the most frequently used one is the electrocardiographic $\mathrm{P}$ wave duration and morphology [8]. Also, there are some echocardiographic methods to measure interatrial conduction times by the evaluation

Address for correspondence: Ali Deniz, MD, Hacettepe University, Faculty of Medicine, Department of Cardiology,

Sihhiye/Ankara/Turkey, tel: 0090505396 19 78, e-mail: alideniz78@gmail.com 
of cardiac electromechanical events. The time interval between the electrocardiographic $\mathrm{P}$ wave and the atrial contraction detected by M-mode or Doppler echocardiography is defined as atrial electromechanical delay [9-11]. Atrial mechanical activity can be detected from different atrial regions by tissue Doppler echocardiography with high temporal resolution [12]. Therefore, tissue Doppler echocardiography may be a useful method to evaluate atrial conduction times.

The purpose of this study was to investigate the correlation between intra- and interatrial conduction times measured by tissue Dopper echocardiography and electrophysiological study, and the appropriateness of tissue Doppler echocardiography to evaluate atrial conduction times.

\section{Methods}

\section{Study population}

One hundered and one patients ( 35 males and 66 females with mean age $41 \pm 12$ years) were enrolled in the study who underwent electrophysiological study with the complaint of palpitations or demonstrated supraventricular tachycardia. Physical examination, 12-lead ECG, chest X-ray, laboratory investigations including complete blood count, blood biochemistry and thyroid function tests were routinely performed for each patient. Laboratory investigations were found to be normal in all of the patients. All patients were free of any other diseases except accessory pathways and dual atrioventricular nodal physiology. Electrophysiologic recordings were obtained when the patients were in normal sinus rhythm with heart rate $60-100 \mathrm{bpm}$, and after the procedure of radiofrequency ablation whenever necessary. Echocardiographic measurements were obtained after electrophysiological study completed in the same day. The patients with chronic AF and pacemakers were excluded from the study. Informed consent was obtained from all patients, and the study was approved by the Hospital Ethics Committee.

\section{Electrocardiographic evaluation of atrial conduction}

Standard ECG were taken from all patients with sweeping rate of $25 \mathrm{~mm} / \mathrm{s}$ and amplitude of $1 \mathrm{mV} / \mathrm{cm}$. All ECGs were evaluated by a cardiologist who was blind to conduction times measured by other methods. The values were calculated by taking the average of three consecutive complexes in each lead. The maximum and minimum durations of $P$ waves (Pmax and Pmin, respectively) were detected, and the difference between Pmax and Pmin was defined as $\mathrm{P}$ wave dispersion $(\mathrm{Pd}=\mathrm{Pmax}-\mathrm{Pmin})$.

\section{Echocardiographic evaluation of atrial conduction times}

All echocardiographic examinations were performed with a System Five (GE Vingmed Ultrasound, Horten, Norway) cardiac ultrasound scanner and 2.5-3.5 MHz transducers. All patients were examined in the left lateral and supine positions by precordial M-mode, 2-dimensional, Doppler and tissue Doppler echocardiography. One lead ECG was recorded continuously. Left ventricular end-diastolic, left ventricular end-systolic, and left atrial endsystolic diameters were measured from $\mathrm{M}$-mode in the parasternal long-axis views according to the standards of the American Society of Echocardiography. Left ventricular ejection fraction was measured from M-mode. Left atrial area was measured by tracing the maximum area of the left atrium during systole in apical 4-chamber view. Left ventricular mass was determined by Teichholz Formula in each subject. Left ventricular diastolic function was evaluated by mitral inflow velocities namely $\mathrm{E}$ peak and A peak, $\mathrm{E} / \mathrm{A}$ ratio and also by deceleration time of the $\mathrm{E}$ wave and isovolumic relaxation time. Tissue Doppler echocardiography was performed by transducer frequencies of 3.5-4.0 MHz, adjusting the spectral pulsed Doppler signal filters until a Nyquist limit of $15-20 \mathrm{~cm} / \mathrm{s}$ and using the minimal optimal gain. The monitor sweep speed was set at $50-100 \mathrm{~mm} / \mathrm{s}$ to optimize the spectral display of myocardial velocities. In apical 4-chamber view, the pulsed Doppler sample volume was subsequently placed at the level of left ventricular lateral mitral annulus, septal mitral annulus, and right ventricular tricuspid annulus. Tissue Doppler pattern was characterized by a positive (i.e., above baseline) myocardial systolic wave (S) and two negative (i.e., below baseline) diastolic waves early (E) and atrial (A). Every effort was made to align the pulsed wave cursor that the Doppler angle of incidence was as close to 0 as possible to the direction of these walls. Time intervals from the onset of P-wave on surface ECG to the beginning of A-wave (PA) representing atrial electromechanical delay were obtained from lateral mitral annulus, septal mitral annulus, and right ventricular (RV) tricuspid annulus and named as lateral PA, septal PA, and RV PA, respectively. The measurement of PA interval is shown in Figure 1. The timing of mechanical activation of each reference point, namely, lateral mitral, septal mitral, and RV tricuspid annuli depends on the distances of these points to sinus node; that 


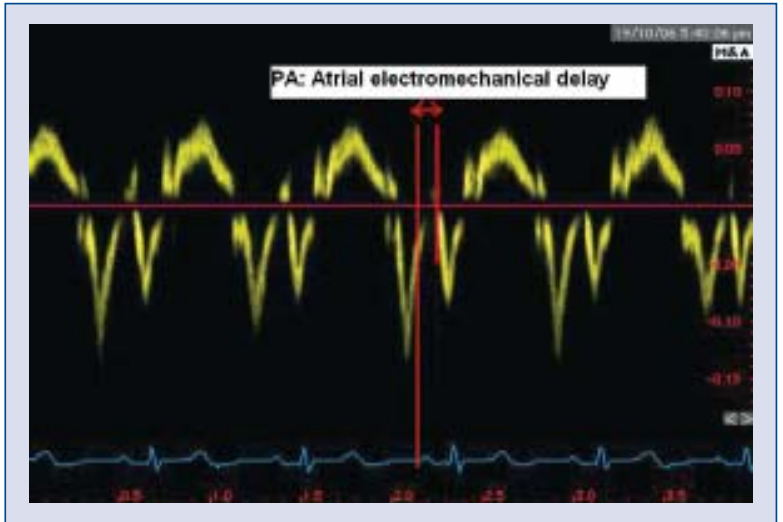

Figure 1. Measurement of atrial electromechanical delay (PA interval) by tissue Doppler echocardiography.

is, the RV tricuspid annulus is the earliest and lateral mitral annulus is the latest points to be activated by the impulse arising from the sinus node. Therefore, it is hypothesized that the difference between any two reference points reflects the conduction time (mechanical delay) between these two points:

- the difference between septal PA and RV PA was defined as intra-right atrial conduction time (IRCT-echo) (septal PA-RV PA);

- the difference between lateral PA and septal PA was defined as intra-left atrial conduction time (ILCT-echo) (lateral PA-septal PA);

- the difference between lateral PA and RV PA was defined as interatrial conduction time (IACT-echo) (lateral PA-RV PA).

\section{Electrophysiologic evaluation of atrial conduction times}

All patients enrolled in the study underwent electrophysiologic study after $8 \mathrm{~h}$ of fasting. Multielectrode catheters were positioned in high right atrium (HRA) at the junction of superior vena cava and right atrium in the region of sinus node, His region, RV apex (RVA) and coronary sinus (CS). The intracardiac electrograms were recorded with a computer containing Prucka CardioLab EP System (GE Medical Systems, Milwaukee, WI, USA) software. After the diagnostic electrophysiological study, radiofrequency ablation was performed if needed. The atrial conduction times were measured from intracardiac electrograms with the sweeping velocity of $400 \mathrm{~mm} / \mathrm{s}$. Electrophysiologic IRCT (IRCT-eps) was measured from the beginning of atrial electrogram in HRA to the beginning of atrial electrogram in His electrode. Electrophysiologic ILCT (ILCT-eps) was measured as the difference between atrial electrograms in proximal and distal

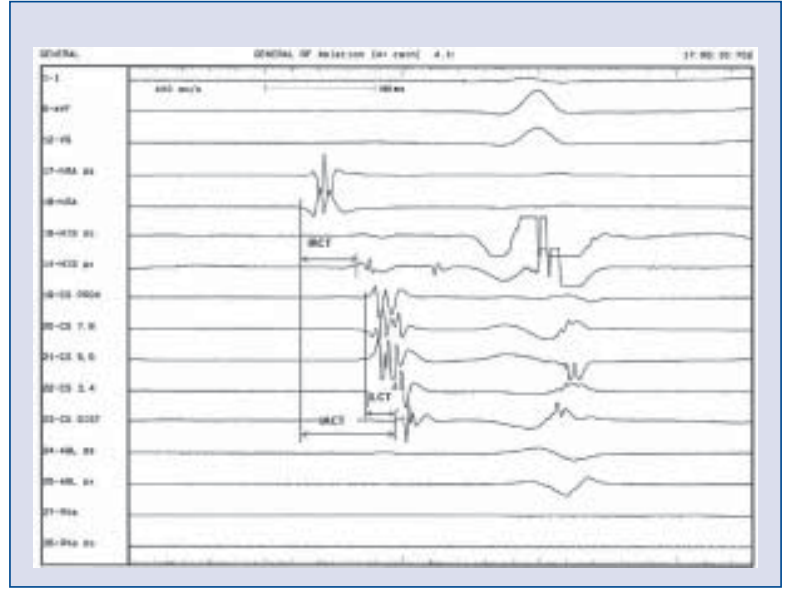

Figure 2. Measurement of interatrial conduction time (IACT), intra-rightatrial conduction time (IRCT) and intra-leftatrial conduction time (ILCT) by electrophysiological study. The time intervals between atrial electrograms of high right atrial and His recordings, proximal and distal coronary sinus recordings, and high right atrium and distal coronary sinus recordings give IRCT, ILCT and IACT, respectively.

CS electrodes. Electrophysiologic IACT (IACT-eps) was defined as the time difference between the atrial electrograms in HRA and distal CS (Fig. 2) [13].

\section{Statistical analysis}

Distribution of data was assessed by using onesample Kolmogorov-Smirnov test. Continuous variables with normal distribution were expressed as mean $\pm \mathrm{SD}$, variables with skew distribution are expressed as median (minimum-maximum), categorical variables are expressed as percentage. For comparison of categorical variables or percentages we used Fisher's exact and $\chi^{2}$ tests. Differences between numeric variables were tested with Student's $t$-test or Mann-Whitney U-test. Correlation was tested with Pearson correlation coefficient. Bland-Altman analysis was done to measure the agreement between ILCT-eps and ILCT-echo. A p value below 0.05 was considered as statistically significant.

\section{Results}

Thirty-five male and 66 female patients were included in the study. The mean age of the patients was $41 \pm 12$ years. Fifty-four female patients underwent radiofrequency ablation with the diagnosis of atrioventricular nodal reentrant tachycardia, the remaining 12 female patients showed normal electrophysiologic findings. Eleven male patients showed 
normal electrophysiologic findings, 24 male patients underwent successful radiofrequency ablation with the diagnosis of atrioventricular nodal reentrant tachycardia $(\mathrm{n}=17)$ and atrioventricular rentrant tachycardia $(\mathrm{n}=7)$. Patient characteristics, ECG, echocardiographic and electrophysiological findings are presented in Table 1. ILCT, IRCT and IACT detected by tissue Doppler echocardiography and electrophysiological study are shown in Table 2.

IACT-echo and IACT-eps showed a weak $(\mathrm{r}=$ $=0.308 ; \mathrm{p}=0.002)$, ILCT-echo and ILCT-eps showed a moderate positive correlation $(r=0.652$; $\mathrm{p}<0.001$ ) (Table 2, Fig. 3). Bland-Altman plot was shown in Figure 4 to evaluate the agreement between ILCT-eps and ILCT-echo. The maximum and minimum $\mathrm{P}$ waves and $\mathrm{P}$ wave dispersion were 98 $\pm 10 \mathrm{~ms}, 53 \pm 9 \mathrm{~ms}$, and $46 \pm 8 \mathrm{~ms}$, respectively. Age $(r=0.246 ; p=0.013$ for ILCT-echo, $r=0.299$; $\mathrm{p}=0.002$ for ILCT-eps), $\mathrm{P}$ wave dispersion $(\mathrm{r}=$ $=0.466 ; \mathrm{p}<0.001$ for ILCT-echo, $\mathrm{r}=0.333 ; \mathrm{p}=$ $=0.001$ for ILCT-eps) and left atrium diameter $(r=0.236 ; p=0.017$ for ILCT-echo, $r=0.202$; $\mathrm{p}=0.043$ for ILCT-eps) showed weak positive, and ejection fraction $(r=-0.220 ; p=0.027$ for ILCT-echo, $r=-0.254 ; \mathrm{p}=0.010$ for ILCT-eps) showed weak negative correlation with both ILCT-echo and ILCT-eps (Fig. 5). There was no prominent relationship between diastolic function parameters determined by mitral inflow velocities and atrial conduction times.

\section{Discussion}

The main finding of our study is the demonstration of moderate correlation between ILCT-echo and ILCT-eps, and weak correlation between IACT-echo and IACT-eps. Since electrophysiological study is an invasive procedure, some sensitive noninvasive markers are needed to evalute atrial conduction times. Tissue Doppler echocardiography may be used for this purpose because of its high temporal and spatial resolution. We found that ILCT detected by tissue Doppler echocardiography was higher in patients with lone paroxysmal AF compared to healthy control group [14]. There was no statistically significant difference in intra-right atrial and interatrial conduction times between the groups, and only ILCT was detected as an independent marker of paroxysmal AF in that study [14]. Merckx et al. [15] demonstrated that tissue Doppler echocardiography can be used to measure total duration of atrial electrical activation which is valuable to detect patients with risk of AF development. In another study, left atrial electromechanical
Table 1. Baseline characteristics, echocardiographic, electrocardiographic and electrophysiologic findings of the patients. $\mathrm{AH}$ and $\mathrm{HV}$ intervals are intracardiac measurements.

\begin{tabular}{lc}
\hline Age [years] & $41 \pm 12$ \\
Gender (male/female) [\%] & $35 / 66(34.7 / 65.3)$ \\
Left atrium diameter [cm] & $3 \pm 0,5$ \\
Left atrium area [cm²] & $14.7 \pm 3.0$ \\
LVEDD [cm] & $4.5 \pm 0.4$ \\
LVESD [cm] & $2.9 \pm 0.4$ \\
EF [\%] & $67 \pm 3$ \\
FS [\%] & $37 \pm 3$ \\
E peak [m/s] & $0.68 \pm 0.15$ \\
A peak [m/s] & $0.64 \pm 0.13$ \\
E deceleration time [ms] & $209 \pm 42$ \\
IVRT [ms] & $93 \pm 25$ \\
E/A ratio & $1.1 \pm 0.4$ \\
Posterior wall thickness [mm] & $8 \pm 1$ \\
Septum thickness [mm] & $8 \pm 1$ \\
Left ventricular mass [g] & $153 \pm 37$ \\
PR interval [ms] & $138 \pm 7$ \\
Pmax duration [ms] & $98 \pm 10$ \\
Pmin duration [ms] & $53 \pm 9$ \\
P wave dispersion [ms] & $46 \pm 8$ \\
QRS duration [ms] & $94 \pm 9$ \\
QT interval [ms] & $354 \pm 49$ \\
AH interval [ms] & $98 \pm 18$ \\
HV interval [ms] & $50 \pm 7$ \\
\hline
\end{tabular}

EF - ejection fraction; FS - fractional shortening; IVRT — isovolumic relaxation time; LVEDD - left ventricular enddiastolic diameter; LVESD - left ventricular endsystolic diameter; Pmax duration duration of the longest $P$ wave; Pmin duration - duration of the shortest P wave

Table 2. Correlation of intra-left atrial (ILCT), intra-right atrial (IRCT) and interatrial conduction times (IACT) detected by tissue Doppler echocardiography and electrophysiological study (EPS).

\begin{tabular}{lcccc}
\hline & Tissue Doppler & EPS & $\mathbf{r}$ & $\mathbf{p}$ \\
\hline ILCT $[\mathrm{ms}]$ & $23 \pm 6$ & $24 \pm 8$ & 0.652 & $<0.001$ \\
IRCT $[\mathrm{ms}]$ & $20 \pm 12$ & $36 \pm 18$ & 0.014 & NS \\
IACT $[\mathrm{ms}]$ & $43 \pm 14$ & $61 \pm 20$ & 0.308 & 0.002 \\
\hline
\end{tabular}

conduction time was found to be significantly prolonged in patients with recurring AF after electrical cardioversion, and it has been stated that electromechanical conduction time parameters evaluated by tissue Doppler echocardiography can be used as indicators of atrial conduction time for prediction of sinus rhythm maintenance [16]. 


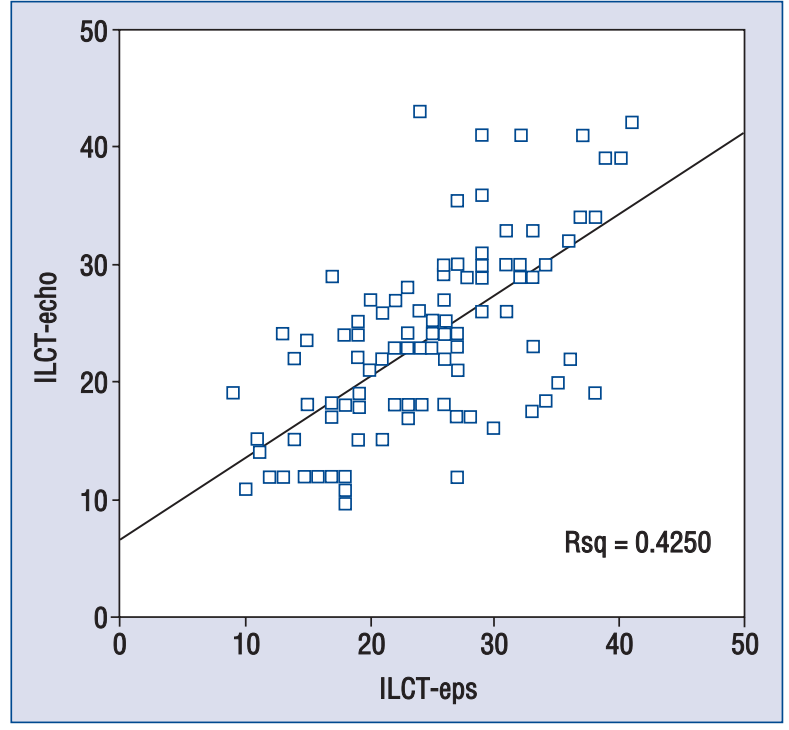

Figure 3. Scatter plot graph showing the correlation between electrophysiological intra-leftatrial conduction time (ILCT-eps) and echocardiographic ILCT (ILCT-echo).

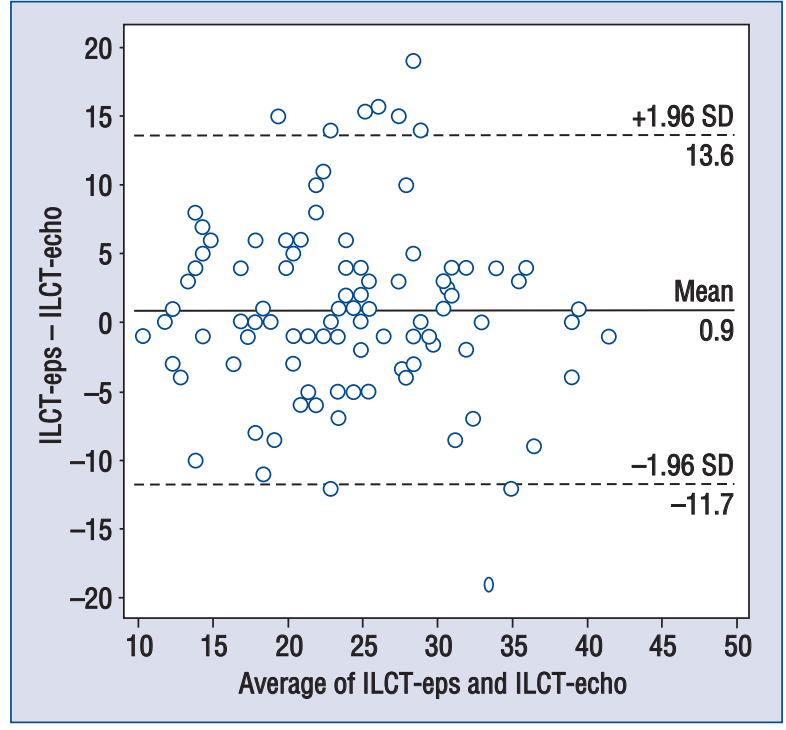

Figure 4. Bland-Altman plot showing the agreement between intra-leftatrial conduction time (ILCT)-eps and ILCT-echo.

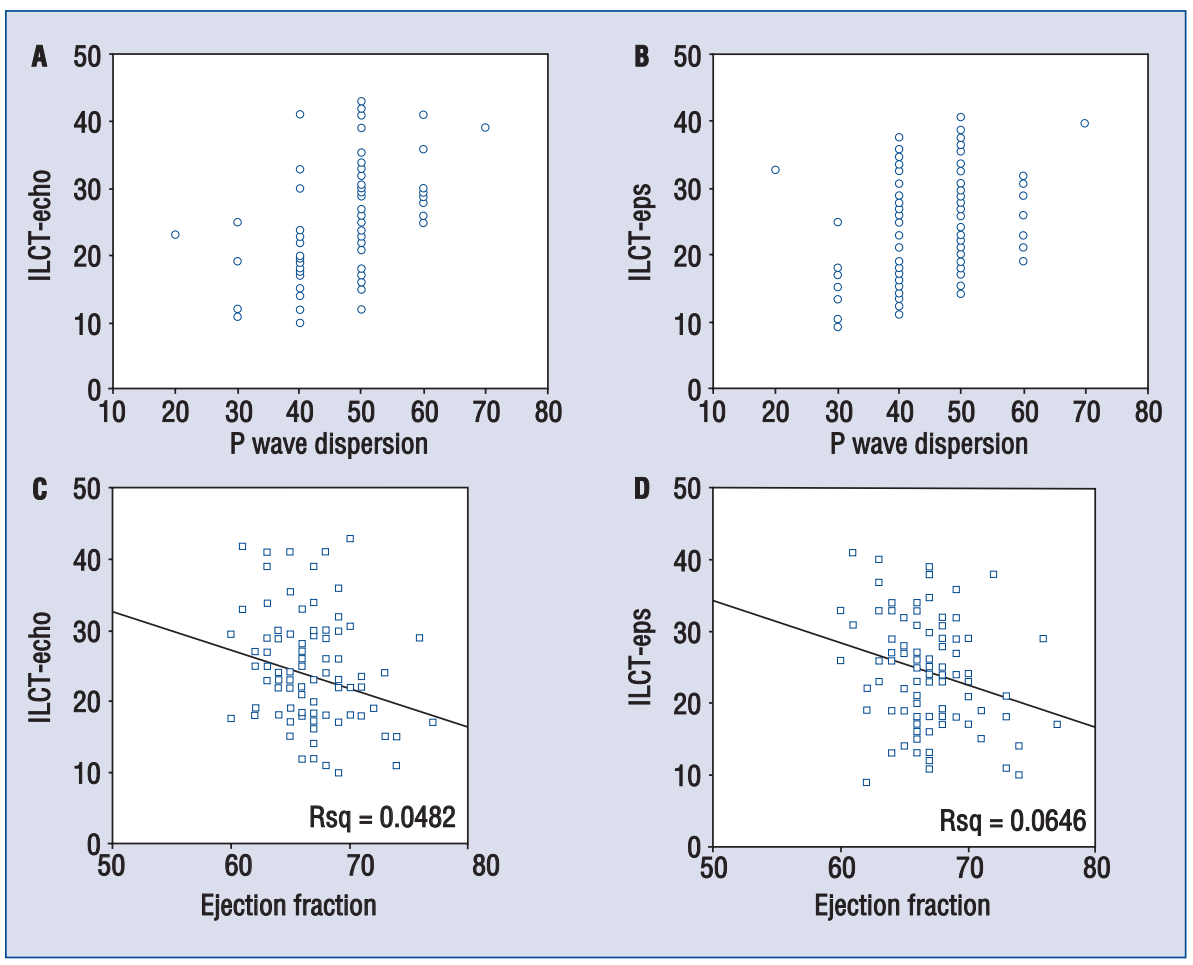

Figure 5. Scatter plot graphs showing the correlation between $\mathrm{P}$ wave dispersion and intra-left atrial conduction time (ILCT)-echo (A), P wave dispersion and ILCT-eps (B), ejection fraction and ILCT-echo (C), and ejection fraction and ILCT-eps (D).

The relationship between left atrial size and $\mathrm{AF}$ has been shown in previous studies [17, 18]. Persistent AF causes electrical and mechanical alter- ations characterized by atrial dilatation and prolonged atrial conduction time [19]. The changes resulting in intra- and interatrial conduction delay 
can prepare convenient atrial substrate for $\mathrm{AF}$. Anisotropy and dispersion of refractory periods resulting from the decrease in electrical connections and depositon of connective tissue between the myocytes cause atrial conduction abnormalities $[8,20]$. Cohen and Scherf [21] was first described the concept of interatrial block. It has been thought that interatrial block may result from the block in Bachmann bundle. Interatrial block has frequently been detected in patients with large left atrium, atrial tachyarrhythmias and sinus and/or atrioventricular nodal dysfunction [4]. According to multiple wavelet hypothesis, the number of wavelets at any time depends on the refractory period, mass and conduction velocity in different parts of the atria. Therefore, delayed conduction increases the number of daughter wavelets and results in continuation of AF [22].

Ozer et al. [23] found that interatrial electromechanical delay is longer in patients with mitral stenosis than in control subjects. In a previous study, we also demonstrated that ILCT and IACT is increased in patients with hypertension with diastolic dysfunction [24]. Interatrial conduction abnormality was shown to be present by tissue Doppler echocardiography in patients with heart failure due to left ventricular systolic dysfunction [25]. Prolonged interatrial conduction time in patients with severe left ventricular systolic dysfunction can affect left atrial remodeling and function [26]. Can et al. [27] evaluated interatrial conduction in patients with scleroderma with the same method. They found that IACT was increased in patients with scleroderma compared to control group.

\section{Limitation of the study}

Main limitation of our study is the measurement of atrial electromechanical delays only in 3 points at the annulus level. Indeed, it has been well known that there are different interatrial connections transmitting the impulse from sinus node to the left atrium. Measurement of atrial electromechanical delay at different atrial regions such as at the base of interatrial septum (that is the region of Bachmann's bundle) might give us more detailed information about the atrial conduction times. HRA and RV tricuspid annulus - which is close to low right atrium instead of high right atrium - are anatomically distinct sites. This may be the reason of the relative weakness of the correlation between IACT-echo and IACT-eps, and the difference between IRCT-echo and IRCT-eps. Instead, CS proximal is close to septal mitral annulus, and CS distal is close to lateral mitral annulus anatomically. Therefore, the correlation is moderate. It is a mat- ter of place of measurements made. Further studies may illuminate this issue. The temporal resolution of tissue Doppler echocardiography is generally satisfactory with sweep speeds of $50-100 \mathrm{~mm} / \mathrm{s}$. During electrophysiological studies, since the beginning of deflections can be obscure and difficult to measure, we used $400 \mathrm{~mm} / \mathrm{s}$ as sweep speed. Different sweep speeds can have some misleading effects on temporal resolution. One other limitation is the inclusion of patients with normal atrial size. Tissue Doppler echocardiographic evaluation of patients with enlarged atria is necessary to confirm the usefulness of this technique.

\section{Conclusions}

To our knowledge, this is the first study comparing tissue Doppler echocardiography and electrophysiological study in the measurement of atrial conduction times. The moderate correlation between ILCT-echo and ILCT-eps shows us that tissue Doppler echocardiography can be used to evaluate atrial conduction time. Atrial conduction times may be probable indirect indicators of atrial substrate. Thus, it is hypothesized that changes in atrial substrate may be evaluated by tissue Doppler echocardiography, and this hypothesis should be tested in future studies. It is not a prospective study to detect the value of tissue Doppler echocardiography to predict AF occurance. Therefore, we need prospective studies to illuminate this issue.

Conflict of interest: none declared

\section{References}

1. Kannel WB, Abbott RD, Savage DD, McNamara PM. Epidemiological features of chronic atrial fibrillation: The Framingham study. N Engl J Med, 1982; 306: 1018-1022.

2. Cosio FG, Palacios J, Vidal JM, Cocina EG, Gomez-Sanchez MA, Tamargo L. Electrophysiologic studies in atrial fibrillation. Slow conduction of premature impulses: A possible manifestation of the background for reentry. Am J Cardiol, 1983; 51: 122-130.

3. Shimizu A, Fukatani M, Tanigawa M, Mori M, Hashiba K. Intra-atrial conduction delay and fragmented atrial activity in patients with paroxysmal atrial fibrillation. Jpn Circ J, 1989; 53: 1023-1030.

4. Bayes de Luna A, Cladellas M, Oter R et al. Interatrial conduction block and retrograde activation of the left atrium and paroxysmal supraventricular tachyarrhythmia. Eur Heart J, 1988; 9: 1112-1118.

5. Daubert JC, Mabo P, Berber V. Atrial tachyarrhythmias associated with high degree interatrial conduction block. Prevention by permanent atrial resynchronization. Eur J Cardiac Pacing Electrophysiol, 1994; 4 (suppl. 1): 35-44.

6. Saksena S, Prakash A, Hill M, Krol RB, Munisf AN, Mathew PP, Mehra R. Prevention of recurrent atrial fibrillation with 
chronic dual site right atrial pacing. J Am Coll Cardiol, 1996; 28: 687-694.

7. Bailin SJ, Johnson WB, Hoyt R. A prospective randomized trial of Bachmann' s bundle pacing for the prevention of atrial fibrillation. J Am Coll Cardiol, 1997; 29 (suppl. A): 74A (abstract).

8. Daubert JC, Pavin D, Jauvert G, Mabo P. Intra- and interatrial conduction delay: Implications for cardiac pacing. PACE, 2004; 27: 507-525.

9. Wang K, Xiao HB, Fujimoto S, Gibson DG. Atrial electromechanical sequence in normal subjects and patients with DDD pacemakers. Heart, 1995; 74: 403-407.

10. Ramsaran EK, Spodick DH. Electromechanical delay in the left atrium as a consequence of interatrial block. Am J Cardiol, 1996; 77: 1132-1134.

11. Barletta G, Del Bene R, Fantini F. The normal sequence of right and left atrial conduction. Ann Noninvasive Electrocardiol, 2001; 6: 222-228.

12. Rein A, O'Donnel CP, Colan SD, Marx GR. Tissue velocity Doppler assessment of atrial and ventricular electromechanical coupling and atrioventricular time intervals in normal subjects. Am J Cardiol, 2003; 92: 1347-1350.

13. Josephson ME. Electrophysiologic investigation: General concepts (chapter 2). In: Josephson ME ed. Clinical cardiac electrophysiology: Techniques and interpretation. $4^{\text {th }}$ Ed. Wolters Kluwer/Lippincott Williams and Wilkins 2008; 20-68.

14. Deniz A, Yavuz B, Aytemir K et al. Intra-left atrial mechanical delay detected by tissue Doppler echocardiography can be a useful marker for paroxysmal atrial fibrillation. Echocardiography, 2009; 26: 779-784.

15. Merckx KL, De Vos CB, Palmans A et al. Atrial activation determined by transthoracic Doppler tissue imaging can be used as an estimate of the total duration of atrial electrical activation. J Am Soc Echocardiogr, 2005; 18: 940-944.

16. Park SM, Kim YH, Choi JI, Pak HN, Kim YH, Shim WJ. Left atrial electromechanical conduction time can predict six-month maintenance of sinus rhythm after electrical cardioversion in patients with atiral fibrillation by Doppler tissue echocardiography. J Am Soc Echocardiogr, 2010; 23: 309-314.
17. Henry WL, Morganroth J, Pearlman AS et al. Relation between echocardiographically determined left atrial size and atrial fibrillation. Circulation, 1976; 53: 273-279.

18. Takahashi N, Imataka K, Seki A, Fujii J. Left atrial enlargement in patients with paroxysmal atrial fibrillation. Jpn Heart J, 1982; 23: 677-683.

19. Pandozi C, Santini M. Update on atrial remodelling owing to rate; does atrial fibrillation always beget atrial fibrillation? Eur Heart J, 2001; 22: 541-553.

20. MS, Dolber PC. Relating extracellular potentials and their derivatives to anisotropic propagation at a microscopic level in human cardiac muscle. Evidence for electrical uncoupling of side-to-side fiber connections with increasing age. Circ Res, 1986; 58: 356-371.

21. Cohen J, Scherf D. Complete interatrial and intra-atrial block (atrial dissociation). Am Heart J, 1965; 70: 24-34.

22. Fuster V, Ryden LE, Cannom DS et al. 2011 ACCF/AHA/HRS focused updates incorporated into the ACC/AHA/ESC 2006 guidelines for the management of patients with atrial fibrillation: a report of the American College of Cardiology Foundation/ /American Heart Association Task Force on Practice Guidelines. J Am Coll Cardiol, 2011; 57: e101-198.

23. Ozer N, Yavuz B, Can I et al. Doppler tissue evaluation of intraatrial and interatrial electromechanical delay and comparison with P-wave dispersion in patients with mitral stenosis. J Am Soc Echocardiogr, 2005; 18: 945-948.

24. Yavuz B, Deniz A, Ertugrul DT et al. A novel echocardiographic marker in hypertensive patients: Is diastolic dysfunctiom associated with atrial electromechanical abnormalities in hypertension? J Clin Hypertens, 2010; 12: 687-692.

25. Van Beeumen K, Duytschaever M, Tavernier R, Van de Veire N, De Sutter J. Intra- and interatrial asynchrony in patients with heart failure. Am J Cardiol, 2007; 99: 79-83.

26. Waggoner AD, Kalathiveetil S, Spence KE, Davila-Roman VG, Fuentes L. Interatrial conduction time and left atrial function in patients with left ventricular systolic dysfunction: Effects of cardiac resynchronization therapy. J Am Soc Echocardiogr, 2009; 22: 472-477.

27. Can I, Onat AM, Aytemir K et al. Assessment of atrial conduction in patients with scleroderma by tissue Doppler echocardiography and P wave dispersion. Cardiology, 2007; 108: 317-321. 